\title{
Enfermagem Avançada - Conceptualização através de Grupos Focais
}

\author{
Carolina Henriques ${ }^{1}$, Paulo Santos ${ }^{2}$ e João Frade ${ }^{3}$
}

\begin{abstract}
${ }^{1}$ Doutorada em Enfermagem, Professora Coordenadora, Departamento de Ciências de Enfermagem, Center for Innovative Care and Health Technology (ciTechCare), Politécnico de Leiria, Portugal | carolina.henriques@ipleiria.pt | https://orcid.org/0000-0002-0904-8057

${ }^{2}$ Doutorado em Enfermagem, Professor Adjunto, Center for Interdisciplinary Research in Health (CIIS), Universidade Católica Portuguesa, Lisboa, Portugal | paulofigueiredosantos@gmail.com| http://orcid.org/0000-0001-7409-1011

${ }^{3}$ Doutorado em Saúde Pública, Professor Adjunto, Departamento de Ciências de Enfermagem, Center for Innovative Care and Health Technology (ciTechCare), Politécnico de Leiria, Portugal | joao.frade@ipleiria.pt | https://orcid.org/0000-0002-4947-1052
\end{abstract}

Resumo: A Enfermagem Avançada é um conceito recente em Portugal, tendo os enfermeiros portugueses se apropriado dele devido ao seu desenvolvimento à evolução da ciência de enfermagem em Portugal. Conhecermos de que forma os enfermeiros conceptualizam o conceito de Enfermagem Avançada é o principal objetivo deste estudo, sendo que, neste artigo procuraremos enfatizar os aspetos metodológicos inerentes à realização de grupos focais. Concluímos que os enfermeiros participantes no estudo conceptualizam o conceito de Enfermagem Avançada assente nos pilares da Investigação, da Conceptualização dos Cuidados, do Processo de Enfermagem, do Desenvolvimento Profissional e da Gestão de Serviços/Instituições. A utilização da metodologia de colheita e análise dos dados no âmbito dos grupos focais mostrou-se bastante adequada, permitindo-nos atingir os objetivos do estudo, tal como, o acesso a interações focadas face ao objetivo do estudo.

Palavras-chave: Enfermagem Avançada; Focus Group; Investigação Qualitativa; Metodologia.

\section{Advanced Nursing - Conceptualization trough Focus Groups}

\begin{abstract}
Advanced Nursing practice is a recent concept in Portugal, leading to Portuguese nurses in its appropriation contributing in professional development and the evolution of nursing science in Portugal. To understand how nurses conceptualize advanced nursing practice, is the main objective of this study, in which trough this article, we seek to emphasize the methodological aspects inherent to the realization of focus groups. We conclude that the nurses participating in the study, conceptualize advanced nursing practice, based on the research, care conceptualization, nursing process methods, professional development and management of hospital wards / health institutions. The use of focus groups research technique, in gathering data and its analysis, proved to be adequate, allowing us to meet the objectives of the study, such as, access to focused interactions in relation to the objective of the study.
\end{abstract}

Keywords: Advanced Nursing Practice; Focus Group; Qualitative Research; Methodology.

\section{Introdução}

O desenvolvimento da Enfermagem como disciplina integra duas dimensões principais: a ciência de enfermagem que promove o desenvolvimento de conhecimentos em enfermagem e a disseminação do conhecimento; e a profissão propriamente dita, que têm uma relação mútua e recíproca com a ciência de enfermagem, não existindo uma sem a outra (Fawcet \& Desanto - Madeya, 2005).

O termo de Enfermagem Avançada surge descrito na literatura em 1980. No entanto, não existe uma definição consensual de enfermagem avançada, já que existem algumas barreiras políticas quanto à representação social do enfermeiro e à natureza das suas intervenções nos contextos clínicos de diferentes países. Implica a extensão das funções profissionais com múltiplas interações em diferentes domínios, nomeadamente a prática clínica, educação investigação desenvolvimento profissional e liderança organizacional (Fernandes \& Varela, 2019). 
O conceito de Enfermagem de Prática Avançada para muitos tornou-se sinónimo de trabalho desenvolvido pelo Advanced Practice Nurse ou enfermeiro perito; outros autores vão mais além e entendem a prática de enfermagem avançada como um processo de desenvolvimento profissional contínuo e aquisição de competências. Além disso, torna-se intimamente ligado com o desenvolvimento da prática (Christensen, 2011).

De acordo com o Conselho Internacional de Enfermagem (ICN), um Enfermeiro de Prática Avançada (APN) é um enfermeiro devidamente credenciado, que adquiriu conhecimentos especializados, possui uma elevada capacidade de tomar decisões e competências clínicas para uma prática avançada. $\mathrm{O}$ acesso a funções avançadas é geralmente precedido de horas de prática clínica e uma especialização e/ou formação académica pósgraduada (Lopes, Gomes \& Lobo, 2018).

Neste sentido, e pela importância que o conceito de Enfermagem Avançada adquiriu em Portugal nos últimos anos, associado também ao próprio desenvolvimento da ciência de enfermagem, considera-se relevante conhecermos de que forma os enfermeiros conceptualizam o conceito de Enfermagem Avançada.

\section{Metodologia}

O presente estudo insere-se numa metodologia qualitativa com recurso a dois grupos focais, onde participaram 13 enfermeiros a frequentar formação de 20 ciclo na área científica de enfermagem e decorreu durante o ano de 2020.

Segundo Morgan (1996,1997), o Focus group é uma metodologia que visa a recolha de dados, através da interação do grupo face a uma ou mais problemáticas que sustentam o objetivo da investigação. Desta forma, para este autor, os grupos focais são um método de investigação centrado na recolha de dados; que se baseia na interação e discussão de um grupo de sujeitos face a uma ou mais problemáticas, onde o investigador é um elemento ativo que conduz o grupo de discussão. Para além do referido anteriormente, os grupos focais centram-se na análise e discussão de uma problemática procurando a sua compreensão (Krueger \& Casey, 2009).

Os grupos focais têm sido amplamente utilizados na investigação em enfermagem, porque se por um lado, permitem-nos a exploração do que é significativo para os sujeitos, pedra basilar da ciência de enfermagem, por outro, segundo Silva, Veloso e Keating (2014) potenciam ao investigador a geração de insights em relação à problemática em estudo. Morgan (1996) salienta que, o que é único nos grupos focais prende-se com a possibilidade de chegarmos aos pontos de acordo e desacordo entre os participantes, permitindo ao investigador ir mais além nos processos de compreensão de um determinado fenómeno.

Com o objetivo de conhecer de que forma os enfermeiros conceptualizam o conceito de Enfermagem Avançada, definimos como questão de investigação que centraliza este trabalho: De que forma os enfermeiros, que frequentam uma formação de $2^{\circ}$ ciclo em enfermagem (mestrado) numa instituição de ensino superior da zona centro de Portugal, conceptualizam o conceito de Enfermagem Avançada?

Como referido anteriormente, participaram no estudo 13 enfermeiros selecionados intencionalmente, organizados aleatoriamente em dois grupos, um grupo constituído por 6 enfermeiros e um outro grupo, constituído por 7 enfermeiros. Desta forma a amostra foi intencional (purposive sampling), procurando selecionar os participantes que gerem previsivelmente análises ricas sobre o fenómeno em estudo (Morgan 1997, 1998). Foram tidos em consideração todos os procedimentos formais e éticos inerentes ao processo investigativo.

Partindo da questão de investigação e tendo por base os pressupostos de Bloor et al. (2001), Krueger e Casey (2009) e Morgan (1998), definimos cinco fases inerentes à realização dos grupos focais, nomeadamente: a fase de planeamento, de preparação, de moderação, de análise dos dados e divulgação dos resultados. 


\subsection{Fase de Planeamento}

Nesta primeira fase, os investigadores procuraram responder a algumas questões norteadoras da implementação do projeto de pesquisa e da metodologia. Assim, procurámos responder aos objetivos do estudo, nomeadamente aos aspetos inerentes à realização dos grupos focais e estrutura do guião para a realização dos mesmos. Procurámos ainda nesta primeira fase, clarificar os critérios de inclusão dos participantes, o número máximo de participantes e a definição do número de grupos focais a realizar.

Nesta fase e seguindo os pressupostos de Morgan (1998), optámos por um guião estruturado de desenvolvimento dos grupos focais, de forma a termos claro a nossa questão de investigação e a não divergirmos entre os dois grupos focais com os diferentes grupos participantes. Utilizámos como recurso, implementar a estratégia do "funil" (Morgan, 1997), em que primeiramente questionámos os enfermeiros com uma questão mais genérica e procurámos ir "afunilando" face ao objetivo do estudo.

\subsection{Fase de Preparação}

Segundo Morgan (1998), nesta fase procurámos assegurar o correto recrutamento dos participantes e todas as condições para a realização dos grupos focais. Assim, sinalizámos a população que tínhamos, dentro dos critérios de inclusão, definidos para o estudo. Posteriormente fizemos o recrutamento dos 13 enfermeiros a frequentar uma formação de $2^{\circ}$ ciclo em enfermagem (mestrado em enfermagem) numa instituição de ensino superior da zona centro de Portugal, através do contacto intencional com os mesmos.

O contacto intencional fez-se através do envio de um convite eletrónico (email) para participar na investigação. Após esta primeira abordagem e aceitação por parte dos participantes, duas semanas antes, enviámos um novo email de confirmação de participação no estudo. Os participantes que constituíram os grupos focais foram distribuídos aleatoriamente por dois grupos, para que posteriormente, procurássemos definir uma data para a realização do grupo focal em cada um dos grupos, tal como a forma mais cómoda para os participantes para a sua realização. Os participantes foram ainda informados por escrito sobre os objetivos do estudo e as regras de organização dos grupos focais, nomeadamente organização e tempo de duração.

Tendo sido consensual que o uso de meios telemáticos seria a forma mais cómoda para a realização dos grupos focais, seguindo os pressupostos de Morgan (1998), os participantes no dia anterior à realização do focus group, foram contactados telefonicamente a agradecer a sua disponibilidade para a realização do estudo, tal como procedemos ao envio do link de acesso ao grupo focal.

\subsection{Fase de Moderação}

Concluídas as fases anteriores, os grupos focais decorreram nas datas e horas previamente agendadas. Durante o grupo focal, somente um investigador fez a moderação dos dois grupos focais, no sentido de manter, o mais possível as condições previamente definidas para a sua realização e adotar o mesmo procedimento em ambos os grupos. Para além disso, entendemos que na escolha do moderador, o mesmo deveria ter facilidade na comunicação, capacidade de questionar e ouvir, tal como promover autorrevelação entre os participantes (Krueger \& Casey, 2009).

Os grupos focais decorreram através de meios telemáticos, tendo sido gravados, para posterior transcrição. Ambos os grupos focais demoraram em média cerca de 60 minutos, o que vai ao encontro do referenciado por Morgan (1996) em que os grupos focais podem ter a duração de 120 minutos. Durante esta etapa, o moderador para além de guiar os grupos focais, tomou nota das expressões faciais, gestos e tom de voz dos participantes. Esta atividade relativa às notas de campo, poderia ter sido realizada por um (co)moderador, o que possivelmente seria mais benéfico, o que nesta investigação não foi possível de realizar. 


\subsection{Fase de Análise e Interpretação dos Dados}

Antes da análise dos dados propriamente dita, os grupos focais previamente gravados, foram transcritos integralmente, tendo este processo demorado cerca de cinco horas, 0 que vai ao encontro de Bloor et al. (2001), Morgan (1997) e Stewart et al. (2007), que nos referem que o processo de transcrição pode demorar oito vezes o tempo de gravação. Posteriormente procedeu-se à fase da análise e interpretação, onde de forma sistemática se recorreu à indução analítica (Znaniecki, 1934, Bloor et al., 2001; Stewart et al., 2007).

Znaniecki (1934), autor de referência da indução analítica, faz a diferenciação clara entre indução analítica e indução enumerativa. A indução analítica, método qualitativo de colheita e análise de dados, refere-se a um processo lógico de passagem do concreto ao abstrato, procurando a identificação das características centrais de um determinado fenómeno. Procurámos ir ao encontro dos pressupostos metodológicos de Znaniecki (1934), Bloor et al. (2001) e Stewart et al. (2007), atendendo às seguintes etapas: a) definição genérica do fenómeno a estudar; b) formulação de explicação hipotética provisória do fenómeno, com possíveis interpretações face ao mesmo; c) confrontação do observado e retirado dos grupos focais, com o fenómeno estudado, à luz de hipóteses indutivas, com o objetivo de se determinar se a/as hipótese(s) se enquadra(m) nos factos, isto é, se é coerente com os factos recolhidos; d) reformulação das proposições conceptuais/hipóteses indutivas, caso não haja correspondência aos factos; e) construção da certeza provável após se ter examinado um pequeno número de casos (a descoberta de casos negativos debilita a explicação e requer nova reformulação hipotética); f) retomase o processo de exame de casos, a redefinição do fenómeno e a reformulação do quadro conceptual e das hipóteses, sempre que não foi possível uma explicação universal. Este processo decorreu manualmente.

Assim, emergiram da reflexão falada dos nossos participantes, macrocategorias e micro/subcategorias, tendo nós procurado compilar os extratos do texto associados, nunca perdendo o contexto de onde os extratos são retirados, tal como as respetivas notas de campo (Tabela 1).

Tabela 1. Indução Analítica face ao fenómeno estudado.

\begin{tabular}{|c|c|c|c|}
\hline Fenómeno & Macrocategorias & Micro/Subcategorias & Exemplo de Extratos \\
\hline \multirow{5}{*}{$\begin{array}{l}\text { Enfermage } \\
\text { m } \\
\text { Avançada }\end{array}$} & Investigação & $\begin{array}{c}\text { Prática baseada na } \\
\text { evidência }\end{array}$ & $\begin{array}{c}\text { BC - "Enfermeiros Peritos com um } \\
\text { pensamento crítico e constante, ou } \\
\text { seja, enfermeiros dados à } \\
\text { investigação..." [gesticula com } \\
\text { conviccão] }\end{array}$ \\
\hline & $\begin{array}{l}\text { Conceptualizaç } \\
\text { ão dos } \\
\text { Cuidados }\end{array}$ & $\begin{array}{l}\text { Modelos teóricos de } \\
\text { enfermagem }\end{array}$ & $\begin{array}{c}\text { BC - “...(re)conceptualização dos } \\
\text { cuidados de enfermagem e à } \\
\text { compreensão mais alargada dos } \\
\text { problemas de enfermagem.” } \\
\text { CT - “....adquirir, desenvolver e } \\
\text { aperfeiçoar conhecimentos e } \\
\text { competências baseadas em } \\
\text { modelos e teorias científicas, ou } \\
\text { mesmo, quadros de referência } \\
\text { (conceitos, pressupostos, valores)" } \\
\text { [aponta com o dedo] }\end{array}$ \\
\hline & & $\begin{array}{l}\text { Compreensão das } \\
\text { vivências }\end{array}$ & $\begin{array}{c}\text { JD - “...compreende as situações } \\
\text { vivenciadas pelo outro e com base } \\
\text { nelas desenvolve eficazmente o } \\
\text { processo de enfermagem." }\end{array}$ \\
\hline & & $\begin{array}{l}\text { Desenvolvimento } \\
\text { de diagnósticos de } \\
\text { enfermagem }\end{array}$ & $\begin{array}{l}\mathrm{PR} \text { - "elabora com facilidade } \\
\text { diagnósticos de enfermagem e } \\
\text { reflete sobre eles..." }\end{array}$ \\
\hline & $\begin{array}{l}\text { Processo de } \\
\text { Enfermagem }\end{array}$ & Resultados sensíveis & $\begin{array}{c}\text { CG - “...nível elevado de } \\
\text { conhecimento académico, } \\
\text { sedimenta o conhecimento e } \\
\text { transfere-o para a prática, } \\
\text { produzindo assim resultados } \\
\text { sensíveis às nossas intervenções." } \\
\text { [gesticula com convicção] }\end{array}$ \\
\hline
\end{tabular}




\begin{tabular}{|c|c|c|c|}
\hline Fenómeno & Macrocategorias & Micro/Subcategorias & Exemplo de Extratos \\
\hline & \multirow{4}{*}{$\begin{array}{l}\text { Desenvolviment } \\
\text { o Profissional }\end{array}$} & $\begin{array}{l}\text { Enfermeiros } \\
\text { especialistas }\end{array}$ & $\begin{array}{c}\mathrm{DB}-\text { "...os enfermeiros } \\
\text { especialistas, já se unem para criar } \\
\text { protocolos que fazem parte dos } \\
\text { padrões de qualidade..." }\end{array}$ \\
\hline & & $\begin{array}{l}\text { Ampla gama de } \\
\text { conhecimentos }\end{array}$ & $\begin{array}{c}\text { AS - “...bagagem educacional } \\
\text { completa e de excelência e que } \\
\text { aplica a mesma..." }\end{array}$ \\
\hline & & $\begin{array}{l}\text { Educação e formação } \\
\text { pós-graduada em } \\
\text { enfermagem }\end{array}$ & $\begin{array}{c}\text { AM - "Os enfermeiros devem } \\
\text { constantemente atualizar os seus } \\
\text { conhecimentos e investir em } \\
\text { formação" [bate com a mão na } \\
\text { mesa] }\end{array}$ \\
\hline & & $\begin{array}{l}\text { Desenvolvimento } \\
\text { ético e } \\
\text { profissional }\end{array}$ & $\begin{array}{c}\text { CA - “...é capaz de desenvolver } \\
\text { habilidades complexas de tomada } \\
\text { de decisão." }\end{array}$ \\
\hline & \multirow{2}{*}{$\begin{array}{c}\text { Gestão de } \\
\text { Serviços/Institui } \\
\text { ções }\end{array}$} & Liderança & $\begin{array}{c}\text { FS - "É autónomo, com } \\
\text { capacidade de liderança e } \\
\text { gestão..." }\end{array}$ \\
\hline & & Melhoria Contínua & $\begin{array}{c}\text { AC - "Gere o serviço e implementa } \\
\text { melhorias de forma continua..." }\end{array}$ \\
\hline
\end{tabular}

Terminada esta fase, os investigadores que utilizam a metodologia dos grupos focais devem discutir os resultados. Antes da discussão propriamente dita, procedemos à devolução e divulgação dos resultados aos participantes, devolvendo não só as transcrições integrais como a organização dos achados que resultaram do processo de indução analítica. Após a aceitação e concordância por parte dos participantes, procedemos à discussão dos achados, nunca perdendo de vista o objetivo do estudo e norteados pela premissa que os grupos focais se destinam a investigações exploratórias, de natureza qualitativa, em que o foco é a busca da compreensão sobre determinado fenómeno.

\section{Resultados e Discussão}

Dos resultados por nós obtidos, verificámos que os enfermeiros participantes neste estudo conceptualizam o conceito de Enfermagem Avançada assente nos pilares da Investigação, da Conceptualização dos Cuidados, do Processo de Enfermagem, do Desenvolvimento Profissional e da Gestão de Serviços/Instituições.

De acordo com o Conselho Internacional de Enfermagem (ICN), um Enfermeiro de Prática Avançada (APN) é um enfermeiro devidamente credenciado que adquiriu conhecimentos especializados, possui uma elevada capacidade de tomar decisões e competências clínicas para uma prática avançada. $\mathrm{O}$ acesso a funções avançadas é geralmente precedido de horas de prática clínica, uma especialização e/ou formação académica pósgraduada (Lopes, Gomes \& Lobo, 2018), o que vai ao encontro do que foi referido pelos nossos participantes, embora estes últimos vão mais longe, entendendo que a enfermagem avançada se centra também na reflexão e (re)conceptualização dos cuidados, na perícia no desenvolvimento do processo de enfermagem e no desenvolvimento profissional.

Um estudo que envolveu 173 líderes de enfermagem na América Latina e Caribe mostranos que apesar destes terem conhecimento do conceito de enfermagem avançada, a maioria desconhecia a legislação e a regulamentação necessária para a sua aplicação (Zug, et al., 2016). 
Ao Enfermeiro de Prática Avançada, é exigido um pensamento crítico constante na construção da disciplina de enfermagem enquanto ciência, na investigação, na re(conceptualização) dos cuidados de enfermagem e na compreensão mais alargada dos problemas de enfermagem, pelo que, consideramos central que os enfermeiros portugueses se possam debruçar sobre o conceito de enfermagem avançada e apropriar das condições de desenvolvimento profissional que estão inerentes ao mesmo.

O International Council of Nurses (ICN) define o enfermeiro de prática avançada como aquele que através de formação adicional adquiriu uma base de conhecimentos equivalente à de perito, possui aptidões complexas na tomada de decisão e competências clínicas para uma prática de enfermagem avançada, a qual pressupõe a extensão e expansão dos limites do âmbito da prática da enfermagem, contribuindo significativamente para o conhecimento em enfermagem, promovendo o avanço da profissão (Schober et al., 2020).

Para Neto, Rewa, Leonello e Oliveira (2018) os mecanismos de regulação da enfermagem de prática avançada devem garantir que o profissional tenha o direito de diagnosticar e a autoridade para prescrever medicamentos e tratamentos e referenciar para outros profissionais de saúde. Segundo o estudo de Maier e Aiken (2016) a enfermagem de prática avançada possibilita o desenvolvimento da enfermagem e dos cuidados de saúde às populações, para isso, estes autores consideram que os países que procuram implementar uma prática de enfermagem avançada devem refletir sobre os ganhos que a enfermagem avançada traz à qualidade e segurança dos cuidados; garantir uma política de diferenciação profissional, tal como uma carreira de enfermagem que reflita o investimento e o desenvolvimento da profissão de enfermagem.

\section{Conclusões}

Esta pesquisa permitiu-nos conhecer e compreender que os enfermeiros participantes no estudo entendem que a Enfermagem Avançada se centra na Investigação, fundamental para o desenvolvimento da ciência de enfermagem e da prática clinica; se centra na conceptualização e reconceptualização dos cuidados de enfermagem, isto é, os enfermeiros de prática avançada conceptualizam os cuidados de enfermagem num movimento contínuo de melhoria dos cuidados que prestam, o que os leva a refletir e a teorizar sobre os mesmos; se centra no Processo de Enfermagem, sendo este norteador da prática de enfermagem, procurando o enfermeiro produzir resultados sensíveis em saúde da sua prática clínica; no desenvolvimento profissional; e na melhoria da gestão de serviços e instituições de saúde.

Para a realização deste estudo, utilizámos os grupos focais e procurámos neste artigo clarificar todas as etapas inerentes à sua realização, seguindo um processo metodológico rigoroso.

Comparando a metodologia dos grupos focais, com outro tipo de metodologias, os grupos focais permitiram-nos colher dados rapidamente, em que neste contexto pandémico se traduziu numa mais-valia, já que houve a possibilidade de aceder em conjunto a um número significativo de participantes, produzindo interações focadas face ao objetivo do estudo, com impactos significativos quer para o grupo de participantes, quer para os próprios investigadores.

Sabemos, pois, que o conceito de Enfermagem Avançada é central para o seu desenvolvimento da ciência de enfermagem e da profissão, pelo que consideramos essencial refletir sobre o conceito, possibilitando-nos também a sua (re)conceptualização. Os resultados por nós encontrados trazem como implicações a necessidade de percebermos quais os aspetos facilitadores e os constrangimentos que os enfermeiros portugueses encontram para a correta implementação de uma enfermagem avançada nos cenários de prática clínica. 


\section{Referências}

Bloor, M., Frankland, J., Thomas, M. \& Robson, K. (2001). Focus groups in social research. London: Sage.

Christensen, M. (2011). Advancing nursing practice: redefining the theoretical and practical integration of Knowledge. Journal Clinical Nursing 20 (5-6), 873-881

Fawcet, J. \& Desanto - Madeya, S. (2005). Contemporary nursing Knowledge: analysis and evaluation of nursing models and theories. 2.ed Philadelphia: F.A. Davis Company

Fernandes, J. \& Varela, D. A. (2019). Enfermagem Avançada. Lisboa: Papa - Letras

Krueger, R. A. \& Casey, M. A. (2009) Focus groups: A pratical guide for applied research (4th Ed.). Thousand Oaks, California: Sage.

Lopes, M., Gomes, B. \& Almada - Lobo (2018). Cuidados de enfermagem especializados como resposta à evolução das necessidades em cuidados de saúde. Ordem dos Enfermeiros INESC - TEC

Maier C. \& Aiken L. (2016). Expanding clinical roles for nurses to realign the global health workforce with population needs: a commentary. Isr J Health Policy Res [Internet], 5(1):21. Available from: https://www.ncbi.nlm.nih.gov/pmc/articles/ PMC4897947/pdf/13584_2016_Article_79.pdf

Morgan, D. L. (1996). Focus group. Annual Review Sociology, 22, 129-152.

Morgan, D. L. (1997). Focus group as qualitative research (2nd ed.). Thousand Oaks, California: Sage.

Morgan, D. L. (1998). Planning focus group. Thousand Oaks, California: Sage.

Neto M. , Rewa T., Leonello V. \& Oliveira M. (2018). Advanced practice nursing: a possibility for Primary Health Care?. Rev Bras Enferm [Internet]. 2018;71(Supl 1):716-21. [Issue Edition: Contributions and challenges of practices in collective health nursing] DOI: http://dx.doi.org/10.1590/0034-7167-2017-0672

Schober, M., Lehwaldt, D., Rogers, M., Steinke, M., Turale, S., Pulcini, J., Roussel, J., Stewart, D. (2020). Guidelines on advanced practice nursing. Geneva: International Council of Nurses.

Silva, I., Veloso, A. \& Keating, J. (2014). Focus group. Considerações teóricas e metodológicas. Revista Lusófona de Educação, 26, 175-190.

Stewart, D. W., Shamdasani, P. N. \& Rook, D. W. (2007). Focus groups: Theory and practice (2nd ed.). Thousand Oaks, California: Sage.

Znaniecki, F. (1934) The Method of Sociology. Farar \& Rinehart, New York.

Zug K., Cassiani S., Pulcini J., Garcia A., Aguirre-Boza F. \& Park J. (2016). Advanced practice nursing in Latin America and the Caribbean: regulation, education and practice. Rev LatinoAm Enfermagem [Internet], 24:e2807. Available from: http://www.scielo.br/pdf/rlae/v24/01041169-rlae-24-02807.pdf 